\title{
Editorial
}

\section{Antibiotic Cycling: Is It Ready for Prime Time?}

\author{
Joseph F. John, Jr, MD
}

\begin{abstract}
The growing, gene-trading, burping, breathing bacteria are suspected to be living control mechanisms in a system of global elemental cycling and gas exchange.

Dorion Sagan, Lynn Margulis Garden of Microbial Delights ${ }^{I}$
\end{abstract}

Cycle (from the Greek kyklos, meaning a ring or circle) implies in English a recurrent sequence of events. Medical imagery abounds with cycles: anovulatory, cardiac, citric acid, endometrial, Krebs, mammary, menstrual, etc. The term cycling, to describe the purposeful alternation of antimicrobials, appeared in the mid-1980s, just about the time of the first substitution trials involving aminoglycosides. ${ }^{2}$ Despite the success of those early aminoglycoside cycling programs, very few experiences with cycling were reported in the 1990s. With the global problem of antimicrobial resistance, particularly in hospitals, ${ }^{3,4}$ antibiotic cycling has reemerged as one of several potential strategies to control antimicrobial selection of resistance. ${ }^{5,6}$ This issue of the Journal is accompanied by a supplement entitled Antibiotic Cycling. My editorial is intended to put the concept of antibiotic cycling and the content of the Supplement into perspective.

The last decade of this millennium has witnessed a growing concern for the problem of antimicrobial resistance and its impending threat to human health (Newsweek. March $23,1994: 46-52$ ). In the last 20 years, pathogens initially susceptible to penicillin $\mathrm{G}$ developed resistance to a range of antimicrobial agents. Nonpathogenic commensals such as the coagulase-negative staphylococci that cover human skin recruited a plethora of resistance genes to plague hospitalized patients, particularly those with plastic devices and prosthetics. Organisms with unpronounceable names overran the cleanest of intensive care units. Some notoriously stubborn bacteria, like the enterococci-already armed with multiple antimicrobial-resistance mechanisms-became resistant to all available agents. ${ }^{3}$ Epidemiologists and clinicians alike wondered if there would ever be an end of the resistance parade. In the short term, would pharmaceutical developers reduce antimicrobial research and development in fear of their products being neutralized even before they had substantial use? What was to be done?

As the 1990s progressed, hospital epidemiologists, infection control coordinators, and hospital pharmacists fought back with new strategies to protect patients from resistant microorganisms, to improve hand washing among healthcare workers, and to maximize appropriate antimicrobial exposure. Pharmaceutical companies adopted molecular technology to identify new antimicrobial targets associated with bacterial adhesion, adaptation, and survival. ${ }^{78} \mathrm{New}$ journals arose dedicated to microbial drug resistance; for example, Microbial Drug Resistance: Mechanism, Epidemiology, and Disease (http://www.liebertpub.com/pubs1.htm). Politicians spoke out against the worsening threat. The government revved its public health engines, and now, as we turn the corner of the new millennium, it is full speed ahead on all these fronts aimed at controlling resistance. Antibiotic cycling is one of the tools being honed to stem the onslaught of emerging and surging resistance in pathogenic microorganisms. What are the data to support the use of cycling? Should we proceed with its implementation?

The major experiments relevant to cycling were done in the mid 1980s, primarily, although not exclusively, in Department of Veterans' Affairs hospitals. ${ }^{9}$ Gerding has now summarized this experience with an eye to the future. ${ }^{10}$ These aminoglycoside substitution programs, although industry supported, were well constructed and informative. They exploited the ability of amikacin to withstand modification by most known aminoglycoside modifying enzymes as a rationale for substituting amikacin for gentamicin or tobramycin. At most participating hospitals, particularly the

From the Division of Allergy, Immunology, and Infectious Disease, the Department of Medicine, University of Medicine and Dentistry of New Jersey, Robert Wood Johnson Medical School, New Brunswick, New Jersey.

Address reprint requests to Joseph F. John, Jr, MD, Division of Allergy, Immunology, and Infectious Disease, 1 RWJ Place 356 MEB, Department of Medicine, University of Medicine and Dentistry of New Jersey, Robert Wood Johnson Medical School, New Brunswick, NJ 08903.

99-ED-151. John JF Jr. Antibiotic cycling: is it ready for prime time? Infect Control Hosp Epidemiol 2000;21:9-11. 


\section{TABLE}

Clinical Scenarios Prompting Consideration of Antibiotic CYCLING

During epidemics caused by pathogens resistant to a narrow spectrum of antimicrobial agents. The agent to be cycled should display a similar spectrum of activity but lack the resistance profile.

For surgical prophylaxis when two classes of agents are considered equivalent, but the utility of one or both agents is reduced due to increased resistance. Cycles may need to be sufficiently long $(\geqslant 1$ year) to elicit measurable benefit.

For introduction of a newly marketed antimicrobial agent with anticipated equivalent or superior efficacy to a currently used agent whose spectrum of activity has decreased.

For use as an alternate agent in a combination regimen (eg, tuberculosis) when the new agent has been shown in vitro to exhibit synergy or improved additive activity.

Minneapolis Veterans' Affairs Medical Center (VAMC), the strategy worked well: replacing gentamicin and tobramycin with amikacin resulted in reduced gentamicin resistance, and, at the Minneapolis VAMC, recycling gentamicin resulted in a subsequent increase in gentamicin resistance. Amikacin resistance remained near baseline during all cycles. ${ }^{11,12}$ To their credit, the investigators conducting these programs were able to include the large numbers of compliant patients needed for proper statistical analysis. Moreover, these trials occurred at a time when the available antibiotics and the high incidence of gram-negative bacillary infections justified aminoglycoside therapy. Lastly, the outcome measures were straightforward: more aminoglycoside resistance or less (not studied were other outcomes, including quality-of-life indicators, persistent carriage of resistant clones, and cost).

Today the same studies would dictate a broader outcome analysis to include the rates of gram-negative bacillary resistance to third-generation cephalosporins, of methicillinresistant Staphylococcus aureus, and of vancomycin-resistant enterococci. In future cycling studies, investigators must consider confounding variables, such as the effectiveness of the infection control program and the effect of community antimicrobial use on hospital resistance. ${ }^{13}$ The so-called ICARE (Intensive Care Antimicrobial Resistance Epidemiology) studies now underway are addressing several potential confounding variables, in particular the infection control measures in intensive care units. ${ }^{14}$

What about the results of antibiotic-cycling studies subsequent to the aminoglycoside substitution programs? As new antimicrobials hit the market in the late 1980s, enthusiasm for cycling waned. The new third-generation cephalosporins, polyfluorinated quinolones, carbapenems, and monobactams were to answer all our antimicrobial needs. Far from it! What in fact happened is that extended resistance in gram-negative bacilli emerged and the problem of resistance in gram-positive cocci escalated, particularly with respect to vancomycin resistance in enterococci and the long-awaited arrival of staphylococcal strains insen- sitive to vancomycin. ${ }^{15}$ As discussed in the Supplement article by Lavin, the prospect of antibiotic cycling has reemerged as a singular strategy to minimize the enormous volume of vancomycin use. ${ }^{16}$

Several additional cycling or substitution studies recently appeared, suggesting that, within the confines of intensive- or special-care areas, short cycles of antimicrobials could modify resistance patterns. Kollef et al studied an antimicrobial change in ventilator-associated pneumonia following cardiac surgery. ${ }^{17} \mathrm{An}$ initial 6-month cycle of standard therapy with ceftazidime was compared to a second 6 month period using ciprofloxacin. The second period saw a significant decrease in pneumonia, primarily due to reduced incidence of antibiotic-resistant gram-negative bacilli. No second round of ceftazidime was evaluated.

Landman et al employed a formulary change to limit use of vancomycin and other agents by increasing use of $\beta$-lactam- $\beta$-lactamase-inhibitor combinations. ${ }^{18}$ The changes resulted in a decrease in the prevalence of methicillin-resistant Staphylococcus aureus and ceftazidimeresistant Klebsiella pneumoniae. There was no attempt to cycle back to the previous formulary. Bradley et al evaluated the cycling of piperacillin-tazobactam after ceftazidime and demonstrated that the ceftazidime cycle showed a significant increase in the rectal carriage of vancomycinresistant enterococci in a hematology-oncology ward whose patients were already heavily colonized $(P=.0001){ }^{19}$ Dominguez et al report in the Supplement a new study of 271 neutropenic patients from a hematology-oncology unit that rotated four antimicrobial combinations and found no difference among regimens with regard to efficacy or resistance. ${ }^{20}$ Thus, even though the data are sparse, antibiotic cycling studies employing effective implementation and well-defined outcomes do suggest some efficacy.

A major consideration for evaluating cycling strategies has come from work done by the pharmacokinetics group in Buffalo. They found that bacteriologic and clinical outcomes depend on the level of antibiotic concentration, expressed as area under the curve over the minimal inhibitory concentration (AUIC). Hyatt and others from the Buffalo group report in the Supplement that, for therapy of infections due to common nosocomial bacteria, an AUIC $\leqslant 125$ was associated with failure. ${ }^{21}$ Clearly variables other than simply the choice of antimicrobial affect clinical outcome and possibly the selective pressure for resistance.

So how should we proceed with implementation of new cycling trials and programs? Certain scenarios may provide a rationale for a given hospital to initiate a cycling program (Table). Once a program for cycling has been selected, certain ideal elements should be included in the program, as outlined in the Supplement by Gerding, ${ }^{10}$ including the ability to distinguish community from nosocomial strains, to establish a stable infection control program, and to type organisms with genetic methods. ${ }^{22}$ While not all elements of an ideal system are needed for the evaluation-or the success - of cycling efforts, both cycling programs and cycling studies will require sizable funding to control confounding variables and to recruit adequate resources. Nevertheless, the current 
expertise in hospital molecular epidemiology could serve these studies well. Government and industry should recognize the unique opportunity to cooperate in funding such studies. It is encouraging that the Centers for Disease Control and Prevention recently issued a request for applications to fund at least two new cycling initiatives.

Caution is needed in designing and implementing future antibiotic-cycling studies. Previously published mathematical projections of the effectiveness of cycling reached pessimistic conclusions, albeit for infections that had no lasting colonization of the host. ${ }^{23}$ Indeed, McGowan, who has studied the epidemiology of antimicrobial resistance for over 30 years, warns of practical pitfalls. ${ }^{24}$ Industry itself remains ambivalent about cycling, for, as Lavin has noted, new antimicrobials may have a relatively short or nonprofitable lifespan. ${ }^{16}$

Perhaps the greatest threat to antibiotic cycling involves the acquisition and assembly of resistance genes in nosocomial pathogens. ${ }^{25}$ Promiscuous transposons contain recombinational hot spots for insertion of integrons containing gene cassettes. Gene cassettes usually contain a single resistance gene that can insert into an array of other resistance genes already present in the transposon. ${ }^{26}$ There is good evidence that these genes, although easily shuffled within transposons, are not easily excised. Moreover, a beneficial effect of reduced antibiotic selective pressure on resistance-gene loss has not been well established. Antibiotic-cycling studies should address the issue of resistance-gene persistence, with attention to careful collection and characterization of pathogens (and perhaps commensals) to determine the rate of resistance-gene persistence in transposons in response to cycling. ${ }^{25}$

Antibiotic resistance has spawned a broad medical and social pessimism. Nevertheless, the response to emerging and reemerging microbial challenges by medical science has been impressive and, in my view, merits a new optimism. Take the example of human immunodeficiency virus infection, an infection once impossible to treat effectively, that currently is relenting to multidrug therapy using specifically targeted antiretroviral agents. Basic research inside and outside of industry will continue to discover novel antimicrobial targets justifying development of classes of antimicrobials. Molecular tools allow us to track resistance organisms closely and at times to limit their spread substantially. Finally, gene therapy promises a new horizon for thinking about how to eliminate chronic infectious diseases, some with widespread antimicrobial resistance, such as tuberculosis, Lyme disease, malaria, herpes simplex, and Helicobacter infection. Antibiotic cycling, while not a panacea for altering the complex factors of antimicrobial selective pressure, presents as a reasonable strategy to test. Substantial time and effort will elapse before we will know if cycling is effective and broadly applicable or if we are simply spinning our wheels.

\section{REFERENCES}

1. Sagan D, Margulis L. Garden of Microbial Delights: A Practical Guide to the Subvisible World. Boston, MA: Harcourt Brace Jovanovich, Inc, Publishers; 1988:74.
2. McGowan JE Jr. Minimizing antimicrobial resistance in hospital bacteria: can switching or cycling drugs help? Infect Control Hosp Epidemiol $1986 ; 7: 573-576$.

3. Wise R, Hart T, Cars O, Streulens M, Helmuth $R$, Huovinen P, et al. Antimicrobial resistance. Is a major threat to public health. $B M J$ 1998;317:609-610.

4. Gerberding JL, McGowan JE Jr, Tenover FC. Emerging nosocomial infections and antimicrobial resistance. Curr Clin Top Infect Dis $1999 ; 19: 83-98$

5. Shlaes DM, Gerding DN, John JF Jr, Craig WA, Bornstein DL, Duncan RA, et al. Society for Healthcare Epidemiology of America and Infectious Diseases Society of America Joint Committee on the Prevention of Antimicrobial Resistance: guidelines for the prevention of antimicrobial resistance in hospitals. Infect Control Hosp Epidemiol 1997;18:275-291.

6. John JF Jr, Fishman NO. Programmatic role of the infectious diseases physician in controlling antimicrobial costs in the hospital. Clin Infect Dis 1997;24:471-485.

7. Service RF. Antibiotics that resist resistance. Science 1995:270:724-727.

8. Knowles DJ. New strategies for antibacterial drug design. Trends Microbiol 1997;5:379-383.

9. Betts RF, Valenti WM, Chapman SW, Chonmaitree T, Mowrer G, Pincus $P$, et al. Five-year surveillance of aminoglycoside usage in a university hospital. Ann Intern Med 1984;100:219-222.

10. Gerding DN. Antimicrobial cycling: lessons learned from the aminoglycoside experience. Infect Control Hosp Epidemiol 2000;21 (suppl):S12-S17.

11. Gerding DN, Larson TA, Hughes RA, Weiler M, Shanholtzer C, Peterson LR. Aminoglycoside resistance and aminoglycoside usage: ten years of experience in one hospital. Antimicrob Agents Chemother 1991;35:1284-1290.

12. Gerding DN, Larson TA. Aminoglycoside resistance in gram-negative bacilli during increased amikacin use. Comparison of experience in 14 United States hospitals with experience in the Minneapolis Veterans Administration Medical Center. Am J Med 1985;79:1-7.

13. Archibald L, Phillips L, Monnet D, McGowan JE Jr, Tenover F, Gaynes $R$. Antimicrobial resistance in isolates from inpatients and outpatients in the United States: increasing importance of the intensive care unit. Clin Infect Dis 1997;24:211-215.

14. Fridkin SK, Edwards JR, Pichette SC, Pryor ER, McGowan JE Jr, Tenover $\mathrm{FC}$, et al. Determinants of vancomycin use in adult intensive care units in 41 Unites States hospitals. Clin Infect Dis 1999;28:1119-1125.

15. Hiramatsu K, Aritaka N, Hanaki H, Kawasaki S, Hosada Y, Hori S, et al. Dissemination in Japanese hospitals of strains of Staphylococcus aureus heterogeneously resistant to vancomycin. Lancet 1997;350:1670-1673.

16. Lavin BS. Antibiotic cycling and marketing into the 21 st century: a perspective from the pharmaceutical industry. Infect Control Hosp Epidemiol 2000;21(suppl):S32-S35.

17. Kollef MH, Vlasnik J, Sharpless L, Pasque C, Murphy D, Fraser V. Scheduled change of antibiotic classes. A strategy to decrease the incidence of ventilator-associated pneumonia. Am J Respir Crit Care Med 1997;156(4 pt 1):1040-1048.

18. Landman D, Chockalingam M, Quale JM. Reduction in the incidence of methicillin-resistant Staphylococcus aureus and ceftazidime-resistant Klebsiella pneumoniae following changes in hospital antibiotic formulary. Clin Infect Dis 1998;28:1062-1066.

19. Bradley SJ, Wilson ALT, Allen MC, Sher HA, Goldstone AH, Scott GM. The control of hyperendemic glycopeptide-resistant Enterococcus spp on a haematology unit by changing antibiotic usage. $J$ Antimicrob Chemother 1999;43:261-266.

20. Dominguez EA, Smith TL, Reed E, Sanders CC, Sanders WE Jr. A pilot study of antibiotic cycling in a hematology-oncology unit. Infect Control Hosp Epidemiol 2000;21(suppl):S4-S8.

21. Hyatt JM, Schentag JJ. Potential role of pharmacokinetics, pharmacodynamics, and computerized databases in controlling bacterial resistance. Infect Control Hosp Epidemiol 2000;21 (suppl):S18-S21.

22. Arbeit RD. Laboratory procedures for the epidemiologic analysis of microorganisms. In: Murray PR, Baron EJ, Pfaller MA, Tenover FC, Yolken RH, eds. Manual of Clinical Microbiology. Washington, DC: American Society for Microbiology Press; 1999:125-137.

23. Bonhoeffer S, Lipsitch M, Levin BR. Evaluating treatment protocols to prevent antibiotic resistance. Proc Natl Acad Sci U S A 1997;94:1210612111.

24. McGowan JE Jr. Strategies for study of the role of cycling on antimicrobial use and resistance. Infect Control Hosp Epidemiol 2000;21 (suppl):S36-S43.

25. John JF Jr, Rice LB. The microbial genetics of antibiotic cycling. Infect Control Hosp Epidemiol 2000;21(suppl):S22-S31.

26. Hall RM, Collis CM. Antibiotic resistance in gram-negative bacteria: the role of gene cassettes and integrons. Drug Resistance Updates 1999;1:109-119. 